\title{
ON FUNCTIONS SUBHARMONIC IN A LIPSCHITZ DOMAIN
}

\author{
JANG-MEI GLORIA WU
}

\begin{abstract}
Let $D$ be a starlike Lipschitz domain in $R^{n}, n>2$. If $w$ is a subharmonic function in $D$ with positive harmonic majorant, then at almost every point on the boundary of $D$ (surface measure), $w$ has radial limit. Results on limits along certain $C^{1}$ curves in general Lipschitz domains are also obtained.
\end{abstract}

1. Main results. The purpose of this note is to prove an analogue of Littlewood's theorem [6] for functions subharmonic on a Lipschitz domain instead of a disk.

Let $D$ be a bounded Lipschitz domain in $R^{n}, n \geqslant 2$; i.e. $\partial D$ can be covered by a finite family of open right cylinders whose bases have positive distances from $\partial D$, and corresponding to each cylinder $L$ there is a coordinate system $x=\left(x_{1}, x^{\prime}\right), x_{1} \in R^{1}$ and $x^{\prime} \in R^{n-1}$, with $x_{1}$-axis parallel to the axis of $L$ and a Lipschitz function $f$ such that

$$
L \cap \partial D=\left\{\left(x_{1}, x^{\prime}\right): x_{1}=f\left(x^{\prime}\right)\right\} \cap L
$$

and

$$
L \cap D=\left\{\left(x_{1}, x^{\prime}\right): x_{1}>f\left(x^{\prime}\right)\right\} \cap L .
$$

Let $M$ be a fixed upper bound of the Lipschitz constants of $f$ s.

THEOREM. If $w$ is a subharmonic function in $D$ with positive harmonic majorant and $L$ is a cylinder with above properties, then at almost all points in $L \cap \partial D, w$ has limits along the line segments parallel to the axis of $L$.

Besides limits along line segments we may also consider limits along certain $C^{1}$ curves. Let $F=\left(F_{i}\right)_{1}^{n-1}$ be a $C^{1}$ function from $L \cap D$ to $R^{n-1}$, and $E$ be a subset of $L \cap \partial D$ with properties (1) $F$ and $\nabla F_{i}$ can be extended continuously to each $x \in E$ through some interior cone of $D$ vertexed at $x$, (2) the normal of $\partial D$ at $x \in E$ is not in the linear span of $\left\{\nabla F_{i}\right\}_{1}^{n-1}$. Then for almost every point $x \in E$, there is a unique nontangential (to $\partial D$ ) level curve of $F$ ending at $x$ [8]. With respect to this family of curves we have the following result.

COROLlary. If $w$ is a subharmonic function in $D$ with positive harmonic

Presented to the Society, January 28, 1977; received by the editors May 4, 1977.

AMS (MOS) subject classifications (1970). Primary 31B05, 31B25.

Key words and phrases. Subharmonic, Green's potential, harmonic measure, Lipschitz domain, limits along curves, Harnack's inequality, maximum principle.

(1) American Mathematical Society 1978 
majorant, then for almost every $x \in E, w$ has limit along the nontangential level curve of $F$ ending at $x$. Especially, if $D$ is starlike, then $w$ has radial limit almost everywhere on $\partial D$.

Limits along more general curves satisfying certain separation properties near $L \cap \partial D$ may also be considered. Details are similar to those in [8, Theorem 1], where results of this kind on the unit disk of the complex plane were obtained.

We notice that the $n-1$ dimensional Hausdorff measure and the harmonic measure have the same null sets on $\partial D,[1]$, an answer to a long-standing problem.

By the Riesz decomposition theorem [3], the function $w$ in the theorem is the difference of two positive harmonic functions minus a Green's potential $(\not \equiv+\infty)$. It is known (see [1] and [4]) that every positive harmonic function in the Lipschitz domain $D$ has nontangential limit almost everywhere on $\partial D$. Thus, to prove the theorem and the corollary it is enough to prove the following.

Proposition. Let $\mu$ be a nonnegative mass distribution on $D$ and $u$ the Green's potential of $\mu$ defined by

$$
u(x)=\int_{D} G(x, y) d \mu(y),
$$

where $G$ is the Green's function of $D$. If $u \neq+\infty$, then (i) at almost all points in $L \cap \partial D, u$ has limit 0 along the segments parallel to the axis of $L$.

(ii) at almost all points in $E$, $u$ has limit 0 along the nontangential level curves of $F$ ending in $E$.

When $n=2$, the proposition was proved in [8]. From now on, we assume $n \geqslant 3$. Our main effort is to give estimates of Green's function in $D$, when the explicit form of Green's function and conformal mappings are not available.

In [9], a sufficient condition on $\mu$ for $u \neq+\infty$ was given and it was proved that under that condition, $u$ has the properties (i) and (ii) with exceptional sets, in most situations, smaller than those described in the proposition. Here we assume $u \neq+\infty$ and impose no other condition on $\mu$.

Other kinds of boundary properties of the subharmonic function $w$ have also been studied. In [7], Silverstein and Wheeden proved that the nontangential $L^{p}(1 \leqslant p<n /(n-2))$ limit of $w$ exists a.e. on $\partial D$. In [2], Diederich proved that if $D$ is Liapunov, then $w$ has mean continuous limit a.e. on $\partial D$.

2. Lemmas and reductions. For a fixed $L$, we consider the coordinate system corresponding to $L$. If $x \in L \cap D$, we use $\tilde{x}$ to denote the point on $L \cap \partial D$ with $\tilde{x}^{\prime}=x^{\prime}$.

Consider a countable covering of $L \cap \partial D$ with open right circular cylinders $S$ contained in $L$ and parallel to $L$, satisfying for each cylinder $S$ in the covering and each $x \in S \cap D$, 
$5 \delta \leqslant$ the $n-1$ dimensional distance from $x^{\prime}$ to

$$
\left\{y^{\prime}: y \text { on the side of } L\right\},
$$

where $\delta$ is the diameter of a base of $S$ and $S \cap D$ is starlike with respect to a point $a$ on the axis of $S$ with property

$$
|a-\tilde{a}| \geqslant 10(1+M) \delta
$$

By a slight adjustment of $S$, if necessary, we may assume $u(a)<+\infty$ because $\{x \in D: u(x)=+\infty\}$ is of capacity zero [3].

We shall construct an auxiliary region $\Omega$. First we assume $\tilde{a}$ is the origin of the coordinate system; thus $a=\left(a_{1}, 0\right)$. For each point $z$ in $\left\{z:\left|z^{\prime}\right|=2 \delta\right\}$ $\cap \partial D$, we associate it with a line segment $l_{z}$ from $z$ to $y$, where $y$ is the point on the intersection of the plane containing $z$ and the $x_{1}$-axis, the plane $\{z$ : $\left.z_{1}=a_{1} / 2\right\}$, and the cylinder $\left|y^{\prime}\right|=3 \delta$. Let $\Omega_{1}$ be the region bounded by the surface $\cup_{z} l_{z}, \partial D$ and $\left\{z: z_{1}=a_{1} / 2\right\}$ and $\Omega_{2}$ be any region with $C^{\infty}$ boundary, starlike about $a$ and satisfying $\left\{z \in L, z_{1}>a_{1} / 2\right.$ and $\left.\left|z^{\prime}\right|<5 \delta\right\}$ $\subseteq \Omega_{2} \subseteq\left\{z \in L, z_{1}>a_{1} / 2\right.$ and $\left.\left|z^{\prime}\right|<6 \delta\right\} . \Omega$ is defined to be $\Omega_{1} \cup \Omega_{2} \cup\{z$ : $z_{1}=a_{1} / 2$ and $\left.\left|z^{\prime}\right|<3 \delta\right\}$. We note that $\Omega$ is a Lipschitz domain contained in $D$, starlike about $a$ and satisfying $S \cap \Omega=S \cap D$.

Let $g$ be the Green's function on $\Omega$. The following lemma can be found in [1]; here, however, a more elementary proof using results by Kellogg [5] instead of Schauder estimates is given.

LEMmA 1. Let $B$ be an open ball in $\Omega$ containing $a$. Then $\partial g(a, x) / \partial x_{1}$ is bounded below on $\Omega \backslash B$.

Proof. We exhaust $\Omega$ by domains $\Omega(k)$ with $C^{\infty}$ boundaries. Moreover we assume $B \subseteq \Omega(k)$ and $\partial \Omega(k)$ is composed of $\partial \Omega_{2} \backslash\left\{z: z_{1}=a_{1} / 2,\left|z^{\prime}\right|<4 \delta\right\}$ and the graph of a $C^{\infty}$ function on $\left\{z \in R^{n-1}:|z|<4 \delta\right\}$. Let $g_{k}$ be the Green's function of $\Omega(k)$ with singularity at $a$ and $B_{0}$ be an open ball in $B$ satisfying $\bar{B}_{0} \subseteq B$ and $a \in B_{0}$. Then $\left\{g_{k}\right\}$ are uniformly bounded on $\Omega(k) \backslash$ $B_{0}$. If for each $x \in \partial B$ we consider the Poisson integral of $g_{k}$ over a sphere in $\cap \Omega(k) \backslash B_{0}$, centered at $x$ and then take $\partial / \partial x_{1}$ under the integral sign, we may conclude that $\left\{\partial g_{k} / \partial x_{1}\right\}$ are uniformly bounded on $\partial B$. Because $\{\partial \Omega(k)\}$ are $C^{\infty},\left\{g_{k}\right\}$ vanish on $\partial \Omega_{2} /\left\{z: z_{1}=a_{1} / 2,\left|z^{\prime}\right|<4 \delta\right\}$, and $\left\{g_{k}\right\}$ are uniformly bounded on $\Omega(k) \backslash B$, following the arguments of Theorems I and III in [5], we conclude that $\left\{\partial g_{k} / \partial x_{1}\right\}$ are uniformly bounded on

$$
\partial \Omega_{2} \backslash\left\{z: z_{1}=a_{1} / 2,\left|z^{\prime}\right|<5 \delta\right\} .
$$

On the rest of $\partial \Omega(k), \partial g_{k} / \partial x_{1}$ has nonnegative boundary values. By noting that $\partial g_{k} / \partial x_{1}$ is harmonic in $\Omega(k) \backslash B$ and letting $k \rightarrow \infty$, we conclude the lemma.

Let $m$ be the $n-1$ dimensional Hausdorff measure on $\partial \Omega$ and $\omega$ be the harmonic measure on $\partial \Omega$ at $a$. Using the fact $\Omega$ is Lipschitz and starlike about $a$ and arguing as in [1], we have the following lemma.

LEMma 2. There is a constant $C$ such that for any $x \in S \cap \partial \Omega$, 


$$
\underset{r \rightarrow 0}{\limsup } \frac{\omega\left(B_{r}\right)}{m\left(B_{r}\right)} \leqslant C \limsup _{r \rightarrow 0} \frac{\partial}{\partial x_{1}} g\left(a,\left(x_{1}+r, x^{\prime}\right)\right)
$$

where $B_{r}=\left\{z \in \partial \Omega,\left|z^{\prime}-x^{\prime}\right|<r\right\}$.

LEMMA 3. Let $A$ be the set of points on $S \cap \partial D$ where $\partial g(a, x) / \partial x_{1}$ has strictly positive nontangential limit. Then $S \cap \partial D \backslash A$ is of measure zero.

Proof. From Lemma 1 and [4], $\partial g(a, x) / \partial x_{1}$ has nontangential limit almost everywhere on $S \cap \partial D$. From Lemma 2 and [1], the points on $S \cap \partial D$ where the nontangential limit is zero form a null set.

To prove the proposition it is sufficient to show the Green's potential $u$ has the desired properties (i) and (ii) in $S \cap \partial D$ and $S \cap E$ respectively. Moreover, we may assume that $\mu$ is concentrated on $S \cap D$.

For each $y \in \Omega, G(x, y)-g(x, y)$ is a positive harmonic function of $x$ on $\Omega$, which vanishes on $S \cap \partial D$. Therefore

$$
\int_{S \cap D}[G(x, y)-g(x, y)] d \mu(y)
$$

is also positive harmonic on $\Omega$ since $u(a)<+\infty$. Let $S_{n}=\{x \in S \cap D$ : $|x-\tilde{x}|<1 / n, \tilde{x} \in S \cap \partial D\}$ and

$$
u_{n}(x)=\int_{S_{n}}[G(x, y)-g(x, y)] d \mu(y) .
$$

Because $\lim _{n \rightarrow \infty} u_{n}(a)=0$, we may choose a subsequence $\left\{u_{n^{\prime}}\right\}$ so that $\Sigma_{n^{\prime}} u_{n^{\prime}}(a)$ is finite. The nontangential limits of $\left\{u_{n^{\prime}}\right\}$ on $S \cap \partial D$ converge to zero almost everywhere. Since $\left\{u_{n}\right\}$ are decreasing, the nontangential limits of $\left\{u_{n}\right\}$ converge to zero almost everywhere. We note that

$$
\int_{S \cap D \backslash S_{n}}[G(x, y)-g(x, y)] d \mu(y)
$$

is zero on $S \cap \partial D$. Therefore $\int_{S \cap D}[G(x, y)-g(x, y)] d \mu(y)$ has nontangential limit zero almost everywhere on $S \cap \partial D$. Let

$$
v(x)=\int_{S \cap D} g(x, y) d \mu(y)
$$

To prove the proposition, it is enough to show the following:

(i)' at almost all points in $S \cap \partial D, v$ has limit zero along the lines parallel to $x_{1}$-axis, and

(ii)' at almost all points in $E \cap S$, $v$ has limit zero along the nontangential level curves of $F$ ending in $E \cap S$.

3. Proof. We shall devote ourselves to the proof of (i)' and (ii)'.

From now on, $c, C, c_{\tilde{x}}$ and $C_{\tilde{x}}$ are strictly positive constants; they can vary from line to line and the last two depend on $\tilde{x}$.

For $q>0$, we denote $\{x \in S \cap D:|x-\tilde{x}|<q\}$ by $S(q)$. If $x \in \Omega$, we use $d(x)$ to denote the distance from $x$ to $\partial \Omega$. From (1), (2), the construction of $\Omega$ and the Lipschitz property, there is a constant $\alpha, 0<\alpha<1$, such that if 
$x \in \Omega,\left|x^{\prime}\right|<\delta$ and $|x-\tilde{x}|<2 \delta$ then

(3)

$$
\alpha|x-\tilde{x}| \leqslant d(x) \text {. }
$$

We recall that for a fixed $y \in \Omega$, the Green's function

$$
g(x, y)=|x-y|^{2-n}-h(x)
$$

where $h(x)$ is the harmonic function in $\Omega$ with boundary value $|y-z|^{2-n}$ at $z \in \partial \Omega$ and that $g(x, y)=g(y, x)$. If $x \in S(2 \delta)$ and $\tilde{x} \in A, A$ as in Lemma 3 , then

$$
c_{\tilde{x}}|x-\tilde{x}| \leqslant g(a, x) \leqslant C_{\tilde{x}}|x-\tilde{x}| .
$$

From now on whenever $x, y$ are in $\Omega$ with $\left|x^{\prime}\right|,\left|y^{\prime}\right|<2 \delta$, we use $s$ and $\sigma$ to denote $|x-\tilde{x}|$ and $|y-\tilde{y}|$ respectively and $\gamma$ to denote $\left|y^{\prime}-x^{\prime}\right|$.

Fix $x \in S(\delta)$ with $\tilde{x} \in A$. We divide $S(\delta)$ into three sets in terms of $y$ as follows.

$$
\begin{aligned}
& S_{1}: y \in S(\delta), \gamma \leqslant s / 2,|\sigma-s| \leqslant s / 2, \\
& S_{2}: y \in S(\delta), \gamma \leqslant s / 2,|\sigma-s|>s / 2, \\
& S_{3}: y \in S(\delta), \gamma>s / 2
\end{aligned}
$$

We want to show

$$
\begin{aligned}
& g(x, y) \leqslant C_{\tilde{x}} g(a, y) s^{-1} \gamma^{2-n} \quad \text { in } S_{1}, \\
& g(x, y) \leqslant C_{\tilde{x}} g(a, y) s^{1-n} \quad \text { in } S_{2}, \\
& g(x, y) \leqslant C_{\tilde{x}} g(a, y) \gamma^{1-n-\rho_{s} \rho} \quad \text { in } S_{3},
\end{aligned}
$$

for some $\rho, 0<\rho<1$, depending on $\Omega$ only.

Let $T=\{y \in \Omega, \gamma \leqslant s / 2$ and $|\sigma-s| \leqslant s / 2\}$. If $y \in T$, from (4) and (5) we have

$$
g(x, y) \leqslant C_{\tilde{x}} g(a, x) s^{-1}|x-y|^{2-n} .
$$

Regarding $g(a, \cdot)$ as harmonic on $\Omega \backslash\{a\}$ and using (3) and Harnack's inequality we have $g(a, x) \leqslant C g(a, y)$. Thus

$$
g(x, y) \leqslant C_{\tilde{x}} g(a, y) s^{-1}|x-y|^{2-n}
$$

which implies (6) because $S_{1} \subseteq T$.

Using the Lipschitz property, we obtain that $|z-x| \geqslant c s$ if $z \in \partial T$. From (9),

$$
g(x, z) \leqslant C_{\tilde{x}} g(a, z) s^{1-n}
$$

for $z \in \partial T$. By the maximum principle,

$$
g(x, y) \leqslant C_{\tilde{x}} g(a, y) s^{1-n}
$$

for $y \in \Omega \backslash(T \cup\{a\})$, which contains $S_{2}$. This proves (7).

Assume $y \in S_{3}$ and $\sigma \leqslant \gamma / 2$. Let $X$ be the point $\left(x_{1}+\gamma, x^{\prime}\right)$. Following the proof of (10) with $x$ replaced by $X$, we obtain

$$
g(X, y) \leqslant C_{\tilde{x}} g(a, y) \gamma^{1-n} .
$$

If $Y$ is any point in $S$ with $Y^{\prime}=y^{\prime}$ and $\gamma \leqslant|Y-\tilde{y}| \leqslant 2 \gamma$, then 


$$
g(Y, y) \leqslant C g(X, y) \leqslant C_{\dot{x}} g(a, y) \gamma^{1-n}
$$

by Harnack's inequality. Let $U$ be the region $\{z \in \Omega, z-\tilde{z}<(1+\alpha / 2) \sigma$ and $\left.\left|z^{\prime}-y^{\prime}\right|<\alpha \sigma / 2\right\}$; recall that $\alpha$ was defined in (3). Let $t$ be the center of the top of $U$, that is, $t=\left(y_{1}+\alpha \sigma / 2, y^{\prime}\right)$. Therefore from (4) we see that for every $z \in \partial U$

$$
g(z, y) \leqslant C \sigma^{2-n} \leqslant C\left[(\alpha \sigma / 2)^{2-n}-(\alpha \sigma)^{2-n}\right] \leqslant C g(t, y) .
$$

Let $U_{0}=U, U_{j}=\left\{z \in \Omega,\left|z^{\prime}-y^{\prime}\right|<2^{j}(\alpha \sigma / 2)\right.$ and $\left.z-\tilde{z}<2^{j}(1+\alpha / 2) \sigma\right\}$ and $t^{j}$ the center of the top of $U_{j}$. Suppose $k$ is the smallest integer $j$ such that $2^{j}(1+\alpha / 2) \sigma \geqslant \gamma$, then

$$
2^{k}(\alpha \sigma / 2) \leqslant 2^{k}(1+\alpha / 2) \sigma / 3 \leqslant 2 \gamma / 3 .
$$

Arguing as in [4, Lemma 2], we may show, by (12) and induction, that if $z \in \partial U_{j}$ and $0 \leqslant j \leqslant k$, then

$$
g(z, y) \leqslant C g\left(t^{j}, y\right)
$$

where $C$ is independent of $k$. From (11) and (14),

$$
g(z, y) \leqslant C_{\tilde{x}} g(a, y) \gamma^{1-n}
$$

for $z \in \partial U_{k}$, thus for $z \in \Omega \backslash U_{k}$. Let $V$ be $\{z \in \Omega,|z-\tilde{x}|<\gamma / 4\}$. Thus $V$ is a subset of $\Omega \backslash U_{k}$ from (13). Let $h$ be the harmonic function on $V$ with boundary value 1 on the spherical part of $\partial V$ and boundary value 0 elsewhere. From [9, Lemma 1], there is a number $\rho, 0<\rho<1$, depending on $\Omega$ only, so that

$$
h(z) \leqslant C|z-\tilde{x}|^{\rho} / \gamma^{\rho}
$$

for every $z \in V$. Because $V \subseteq \Omega \backslash U_{k}$, from (15), (16) and the maximum principle

$$
g(x, y) \leqslant C_{\tilde{x}} g(a, y) \gamma^{1-n-\rho_{S} \rho}
$$

if $s<\gamma / 4$. If $s \geqslant \gamma / 4$, from (15)

$$
g(x, y) \leqslant C_{\dot{x}} g(a, y) \gamma^{1-n} \leqslant C_{\dot{x}} g(a, y) \gamma^{1-n-\rho_{S} \rho} .
$$

We have proved (8) in the case $\sigma \leqslant \gamma / 2$.

Now we assume $y \in S_{3}$ and $\sigma>\gamma / 2$. Let $p$ be the point $\left(x_{1}+\sigma, x^{\prime}\right)$. Following the proof of (6) or (7) with $x$ replaced by $p$, we have

$$
g(p, y) \leqslant C_{\bar{x}} g(a, y) \gamma^{1-n} .
$$

Let $Q=\left\{z \in \Omega: z-\tilde{z}<\sigma+\alpha \gamma / 4\right.$ and $\left.\left|z^{\prime}-y^{\prime}\right|<\alpha \gamma / 2\right\}$ and $q$ be the point $\left(y_{1}+\alpha \gamma / 4, y^{\prime}\right)$. Arguing as the proof of (12), we have $g(z, y) \leqslant$ $C g(q, y)$ for $z \in \partial Q$, also for $z \in \Omega \backslash Q$. By Harnack's inequality we have

$$
g(q, y) \leqslant C g(p, y) .
$$

Hence

$$
g(z, y) \leqslant C_{\bar{x}} g(a, y) \gamma^{1-n}
$$


for $z \in \Omega \backslash Q$. Arguing as in the last paragraph we may obtain (8) from (18). This completes the proof of (8).

The rest of the proof of (i)' is similar to that in [6]. We shall not put in too many details, but we shall give the corresponding references.

Let $\varepsilon(q)=\int_{S(q)} g(a, y) d \mu(y)$ for $q>0$; and let $\Phi(\tilde{x}, t)$ be $\int g(a, y) d \mu(y)$ extended over $\left\{y \in S(q):\left|y^{\prime}-\tilde{x}^{\prime}\right|<t\right\}$ if $\tilde{x} \in S \cap \partial D$. Let $E(q)$ be the set of $\tilde{x}$ on which

$$
\limsup _{t \rightarrow 0} \frac{\Phi(\tilde{x}, t)}{t^{n-1}} \leqslant \sqrt{\varepsilon(q)} .
$$

It is known that the complement of $E(q)$ in $S \cap \partial D$ is of measure $\leqslant C \sqrt{\varepsilon(q)}$, where $C$ is independent of $q[6$, p. 393]. In order to prove (i)', it is enough to show that for each small $q>0$ and for each $\tilde{x} \in E(q) \cap A$,

$$
\lim \sup \int_{S(q)} g(x, y) d \mu(y) \leqslant C_{\tilde{x}} \sqrt{\varepsilon(q)}
$$

as $x \rightarrow \tilde{x}$ along the segment $x^{\prime}=\tilde{x}^{\prime}[6$, p. 392]. From (6), (7) and (8) we have

$$
\begin{aligned}
& \int_{S(q)} g(x, y) d \mu(y) \\
& \quad \leqslant C_{\tilde{x}}\left[\int_{0}^{s} s^{-1} \gamma^{2-n} d \Phi(\gamma)+\int_{0}^{s} s^{1-n} d \Phi(\gamma)+\int_{s}^{C} s^{\rho} \gamma^{1-n-\rho} d \Phi(\gamma)\right]
\end{aligned}
$$

where $\Phi(\gamma)=\Phi(\tilde{x}, \gamma)$. (20) may be obtained by applying (19) to the above integrals. We complete the proof of (i)'.

The main devices of the proof of (ii)' are inequalities corresponding to (6), (7) and (8) on curvilinear cylindrical regions whose sides are $C^{1}$ surfaces instead of cylindrical surfaces. To derive them, first we apply Whitney's extension theorem to replace $F$ by a function in $C^{1}(\bar{\Omega})$; next we apply Harnack's inequality to the three inequalities mentioned above to show the desired ones. Details are similar to those in [8, Theorem 2].

AdDED IN Proof. Since the submitting of this paper, I have learned one by B. Dahlberg, On the existence of radial boundary values for functions subharmonic in a Lipschitz domain. Using fine limit approach, Dahlberg considered limits along a family of line segments whose directions satisfy a Lipschitz condition.

\section{REFERENCES}

1. B. Dahlberg, On estimates of harmonic measure, Arch. Rational Mech. Anal. 65 (1977), 275-288.

2. J. R. Diederich, Natural limits for harmonic and superharmonic functions, Trans. Amer. Math. Soc. 224 (1976), 381-397.

3. L. L. Helms, Introduction to potential theory, Wiley, New York, 1969.

4. R. A. Hunt and R. L. Wheeden, On the boundary values of harmonic functions, Trans. Amer. Math. Soc. 132 (1968), 307-322.

5. O. D. Kellogg, On the derivatives of harmonic functions on the boundary, Trans. Amer. Math. Soc. 33 (1931), 486-510. 
6. J. E. Littlewood, On functions subharmonic in a circle. II, Proc. London Math. Soc. (2) 28 (1928), 383-394.

7. M. L. Silverstein and R. L. Wheeden, Superharmonic functions on Lipschitz domains, Studia Math. 39 (1971), 191-198.

8. J.-M. Wu, Boundary limits of Green's potentials along curves, Studia Math. 60 (1976), 137-144.

9. _ Boundary limits of Green's potentials along curves. II. Lipschitz domains, Studia Math. (to appear).

Department of Mathematics, PURdue University, West Lafayette, Indiana 47907 